\title{
A REMARK ON INVARIANCE OF QUANTUM MARKOV SEMIGROUPS
}

\author{
Veni Choi And Chul Ki Ko
}

\begin{abstract}
In [3, 9], using the theory of noncommutative Dirichlet forms in the sense of Cipriani [6] and the symmetric embedding map, authors constructed the KMS-symmetric Markovian semigroup $\left\{S_{t}\right\}_{t \geq 0}$ on a von Neumann algebra $\mathcal{M}$ with an admissible function $f$ and an operator $x \in \mathcal{M}$. We give a sufficient and necessary condition for $x$ so that the semigroup $\left\{S_{t}\right\}_{t \geq 0}$ acts separately on diagonal and off-diagonal operators with respect to a basis and study some results.
\end{abstract}

\section{Introduction}

A quantum Markov semigroup on the algebra $L(\mathfrak{h})$ of all bounded operators on a complex separable Hilbert space $\mathfrak{h}$ is a semigroup $\left\{S_{t}\right\}_{t>0}$ of completely positive, identity preserving and normal maps on $L(\mathfrak{h})[6]$. Quantum Markov semigroups are the natural generalization of classical Markov semigroups and were introduced in physics to model the decay equilibrium of quantum open systems $[1,2,3,6,7,9,10]$. Many mathematicians and physicists have been interested to the problems whether quantum Markov semigroups on the subalgebra have their extensions on the full algebra $[1,5,8]$. These semigroups acts separately on diagonal and off-diagonal bounded operators with respect to the chosen basis.

Let $\mathcal{M}$ be a von Neumann algebra acting on a complex Hilbert space $\mathcal{H}$ and $\xi_{0}$ be a fixed cyclic and separating vector for $\mathcal{M}$. Let $\Delta$ and $\sigma_{t}$ be the modular operator and the modular group associated with the pair $\left(\mathcal{M}, \xi_{0}\right)$, respectively [4]. Consider the symmetric embedding map:

$$
\begin{aligned}
& i_{0}: \mathcal{M} \longrightarrow \mathcal{H} \\
& i_{0}(A)=\Delta^{1 / 4} A \xi_{0} .
\end{aligned}
$$

In $[3,9]$, using the theory of noncommutative Dirichlet forms in the sense of Cipriani [6], authors constructed the symmetric Markovian semigroup $\left\{T_{t}\right\}_{t \geq 0}$ on the standard forms of the von Neumann algebra $\mathcal{M}$, and by the symmetric

Received August 11, 2007.

2000 Mathematics Subject Classification. 46L55, 37A60.

Key words and phrases. quantum Markov semigroups, diagonal operators, invariant subspaces. 
embedding map $i_{0}$, the KMS-symmetric Markovian semigroup $\left\{S_{t}\right\}_{t>0}$ on $\mathcal{M}$ : $i_{0} \circ S_{t}=T_{t} \circ i_{0}$. Concretely, for a fixed admissible function $f$ and $x \in \mathcal{M}, x=$ $x_{1}+i x_{2}, x_{k}^{*}=x_{k}, k=1,2$ satisfying suitable conditions, they constructed the KMS-symmetric Markovian semigroup $\left\{S_{t}\right\}$ with the generator $G$ on $\mathcal{M}$ :

$$
G(A)=\int\left[G_{1}(A, t)+G_{2}(A, t)\right] f(t) d t,
$$

where

$$
\begin{aligned}
G_{1}(A, t)= & \sigma_{t+i / 2}\left(x_{1}\right) \sigma_{t}\left(x_{1}\right) A+A \sigma_{t}\left(x_{1}\right) \sigma_{t-i / 2}\left(x_{1}\right) \\
& -\sigma_{t+i / 2}\left(x_{1}\right) A \sigma_{t}\left(x_{1}\right)-\sigma_{t}\left(x_{1}\right) A \sigma_{t-i / 2}\left(x_{1}\right)
\end{aligned}
$$

and

$$
\begin{aligned}
G_{2}(A, t)= & \sigma_{t+i / 2}\left(x_{2}\right) \sigma_{t}\left(x_{2}\right) A+A \sigma_{t}\left(x_{2}\right) \sigma_{t-i / 2}\left(x_{2}\right) \\
& -\sigma_{t+i / 2}\left(x_{2}\right) A \sigma_{t}\left(x_{2}\right)-\sigma_{t}\left(x_{2}\right) A \sigma_{t-i / 2}\left(x_{2}\right) .
\end{aligned}
$$

In this paper, we consider the faithful, normal, semifinite trace $\operatorname{Tr}$ on $L(\mathfrak{h})$. The Hilbert-Schmidt class $L^{2}(\mathfrak{h})$ is a Hilbert space with the inner product, $\langle\xi, \eta\rangle=\operatorname{Tr}\left(\xi^{*} \eta\right) . \pi_{L}$ is the faithful, normal representation of $L(\mathfrak{h})$ given by $\pi_{L}(x)=L_{x}, L_{x} \xi=x \xi$ for $\xi \in L^{2}(\mathfrak{h})$. Put $\mathcal{M}=\pi_{L}(L(\mathfrak{h}))$.

Let $\left\{e_{k}\right\}_{k=0}^{\infty}$ be an orthonormal basis of $\mathfrak{h}$. Let $\rho, \omega$ and $\xi_{0}$ be a strictly positive density matrix, the corresponding normal state and the corresponding cyclic and separating vector, respectively:

$$
\begin{aligned}
& \rho=\sum_{k=0}^{\infty} r_{k}\left|e_{k}\right\rangle\left\langle e_{k}\right|, \text { and } r_{k}>0, \sum_{k=0}^{\infty} r_{k}=1, \\
& \omega\left(L_{x}\right)=\operatorname{Tr}(\rho x)=\left\langle\xi_{0}, x \xi_{0}\right\rangle, \\
& \xi_{0}=\rho^{1 / 2}=\sum_{k=0}^{\infty} r_{k}^{1 / 2}\left|e_{k}\right\rangle\left\langle e_{k}\right| .
\end{aligned}
$$

Since $\pi_{L}$ is a monomorphism, we identify $\mathcal{M}=\pi_{L}(L(\mathfrak{h}))$ with $L(\mathfrak{h})$.

The purpose of this paper is to find a sufficient and necessary condition for $x \in \mathcal{M}$ used to construct the semigroup $\left\{S_{t}\right\}_{t \geq 0}$ on $\mathcal{M}$ so that the semigroup acts separately on diagonal and off-diagonal operators with respect to this basis and, as an application, study the KMS-symmetric Markovian semigroup $\left\{S_{t}\right\}_{t>0}$ which is $\rho$-invariant.

This paper is organized as follows. In Section 2, we introduce a necessary terminologies and the generator of KMS-symmetric Markovian semigroup on $\mathcal{M}$ constructed in $[3,9]$. In Section 3 , we give our main results and proofs.

\section{The quantum Markov semigroup}

In this section, we introduce some terminologies and the generator of KMSsymmetric Markovian semigroup on $\mathcal{M}$ constructed in [3, 9].

Let $\mathcal{M}$ be a $\sigma$-finite von Neumann algebra acting on a complex Hilbert space $\mathcal{H}$ with an inner product $\langle\cdot, \cdot\rangle$. Let $\xi_{0} \in \mathcal{H}$ be a cyclic and separating vector 
for $\mathcal{M}$. We use $\Delta$ and $J$ to denote respectively, the modular operator and the modular conjugation associated with the pair $\left(\mathcal{M}, \xi_{0}\right)$. The associated modular automorphism group is denoted by $\sigma_{t}: \sigma_{t}(A)=\Delta^{i t} A \Delta^{-i t}, A \in \mathcal{M}, t \in \mathbb{R}$. The map $j: \mathcal{M} \rightarrow \mathcal{M}^{\prime}$ is the antilinear $*$-isomorphism defined by $j(A)=J A J, A \in$ $\mathcal{M}$, where $\mathcal{M}^{\prime}$ is the commutant of $\mathcal{M}$.

The positive cone $\mathcal{P}$ associated with the pair $\left(\mathcal{M}, \xi_{0}\right)$ is the closure of the set $\left\{A j(A) \xi_{0}: A \in \mathcal{M}\right\} . \mathcal{P}$ can be obtained by the closure of the set $\left\{\Delta^{1 / 4} A^{*} A \xi_{0}\right.$ : $A \in \mathcal{M}\}$ and is self-dual in the sense that

$$
\{\xi \in \mathcal{H}:\langle\xi, \eta\rangle \geq 0, \forall \eta \in \mathcal{P}\}=\mathcal{P} .
$$

For the details we refer [6] and Section 2.5 of [4].

The form $(\mathcal{M}, \mathcal{H}, \mathcal{P}, J)$ is the standard form associated with the pair $\left(\mathcal{M}, \xi_{0}\right)$. The Hilbert space $\mathcal{H}$ is the complexification of the real subspace $\mathcal{H}^{J}:=\{\xi \in$ $\mathcal{H}:\langle\xi, \eta\rangle \in \mathbb{R}, \forall \eta \in \mathcal{P}\}$, whose elements are called $J$-real: $\mathcal{H}=\mathcal{H}^{J} \oplus i \mathcal{H}^{J}$. Such a positive cone $\mathcal{P}$ gives a rise to a structure of ordered Hilbert space on $\mathcal{H}^{J}$ (denoted by $\leq$ ) and an anti-unitary involution $J$ on $\mathcal{H}$ by $J(\xi+i \eta):=$ $\xi-i \eta, \forall \xi, \eta \in \mathcal{H}^{J}$. For $\xi, \eta \in \mathcal{H}^{J}, \xi \leq \eta$ means $\eta-\xi \in \mathcal{P}$. Any $J$-real element $\xi \in \mathcal{H}^{J}$ can be decomposed uniquely as a difference of two orthogonal, positive elements, called the positive and the negative part of $\xi: \xi_{+}, \xi_{-} \in$ $\mathcal{P},, \xi=\xi_{+}-\xi_{-},\left\langle\xi_{+}, \xi_{-}\right\rangle=0$. The order interval $\left\{\eta \in \mathcal{H}: 0 \leq \eta \leq \xi_{0}\right\}$, denoted by $\left[0, \xi_{0}\right]$, is a closed convex subset of $\mathcal{H}$, and we denote the nearest point projection onto $\left[0, \xi_{0}\right]$ by $\eta \rightarrow \eta_{I}$.

Let $\mathcal{M}_{0} \subset \mathcal{M}$ be the $*$-subalgebra of the $\sigma_{t}$-entire analytic elements [4] and $\mathcal{M}_{+}$the subset of positive elements of $\mathcal{M}$. Let $\omega$ be a vector state on $\mathcal{M}$ such that $\omega(A):=\left\langle\xi_{0}, A \xi_{0}\right\rangle, A \in \mathcal{M}$.

Consider the semigroup $\left\{S_{t}\right\}_{t \geq 0}$ of everywhere defined linear maps on $\mathcal{M}$. A semigroup $\left\{S_{t}\right\}_{t \geq 0}$ is said to be $K M S$-symmetric if for all $t \in \mathbb{R}$ and for all $A, B \in \mathcal{M}_{0}$, one has

$$
\omega\left(S_{t}(A) \sigma_{-i / 2}(B)\right)=\omega\left(\sigma_{i / 2}(A) S_{t}(B)\right) .
$$

A semigroup $\left\{S_{t}\right\}_{t \geq 0}$ is said to be real if $S_{t}\left(A^{*}\right)=\left(S_{t}(A)\right)^{*}$ for all $A \in \mathcal{M}$ and for all $t \geq 0$, positive preserving if $S_{t}(A) \in \mathcal{M}_{+}$for all $A \in \mathcal{M}_{+}$and for all $t \geq 0$, sub-Markovian if $0 \leq S_{t}(A) \leq \mathbf{1}$ for all $0 \leq A \leq \mathbf{1}$ and for all $t \geq 0$. A semigroup $\left\{S_{t}\right\}_{t \geq 0}$ is said to be Markovian if $S_{t}$ is positive preserving and $S_{t}(\mathbf{1})=\mathbf{1}$ for all $t \geq 0$.

Next, we consider a complex valued, closed, positive sesquilinear form on some linear manifold of $\mathcal{H}: \mathcal{E}(\cdot, \cdot): D(\mathcal{E}) \times D(\mathcal{E}) \rightarrow \mathbb{C}$ satisfying $\mathcal{E}(\xi, \xi) \geq 0$ for all $\xi \in D(\mathcal{E})$, and also the associated quadratic form: $\mathcal{E}[\cdot]: D(\mathcal{E}) \rightarrow \mathbb{C}$, $\mathcal{E}[\xi]:=\mathcal{E}(\xi, \xi)$. A quadratic form $(\mathcal{E}, D(\mathcal{E}))$ is said to be $J$-real if $J D(\mathcal{E}) \subset D(\mathcal{E})$ and $\mathcal{E}[J \xi]=\mathcal{E}[\xi]$ for any $\xi \in D(\mathcal{E})$, equivalently, $\mathcal{E}(J \xi, J \eta)=\mathcal{E}(\eta, \xi)$ for all $\xi, \eta \in D(\mathcal{E})$.

A $J$-real, real-valued, densely defined quadratic form $(\mathcal{E}, D(\mathcal{E}))$ is called Markovian (with respect to $\xi_{0}$ ) if

$$
\xi \in D(\mathcal{E}) \cap \mathcal{H}^{J} \Rightarrow \xi_{I} \in D(\mathcal{E}), \quad \mathcal{E}\left[\xi_{I}\right] \leq \mathcal{E}[\xi] .
$$


A closed Markovian form is called a Dirichlet form.

For a positive closed form $(\mathcal{E}, D(\mathcal{E}))$, there exists a positive self-adjoint operator $(H, D(H))$ such that

$$
\mathcal{E}(\xi, \eta)=\langle\xi, H \eta\rangle, \xi, \eta \in D(\mathcal{E}),
$$

and a strongly continuous, symmetric semigroup $\left\{T_{t}\right\}_{t \geq 0}, T_{t}=e^{-t H}$. Moreover, when $(\mathcal{E}, D(\mathcal{E}))$ is a Dirichlet form, $H$ is called a Dirichlet operator. For the details, see Section 3.1 of [4].

In [9], the author has used the notion of admissible function to construct Dirichlet forms.

Definition 2.1. An analytic function $f: D \rightarrow \mathbb{C}$ on a domain $D$ containing the strip $\operatorname{Im} z \in[-1 / 4,1 / 4]$ is said to be admissible if the following properties hold:

(a) $f(t) \geq 0$ for all $t \in \mathbb{R}$,

(b) $f(t+i / 4)+f(t-i / 4) \geq 0$ for all $t \in \mathbb{R}$,

(c) there exist $M>0$ and $p>1$ such that the bound

$$
|f(t+i s)| \leq M(1+|t|)^{-p}
$$

holds uniformly in $s \in[-1 / 4,1 / 4]$.

The function $g(t)$ given by

$$
g(t)=\frac{2}{\sqrt{2 \pi}} \int\left(e^{k / 4}+e^{-k / 4}\right)^{-1} e^{-\frac{1}{2} k^{2}} e^{-i k t} d k
$$

is admissible. See the proof of Lemma 3.1 of [9].

Using a fixed admissible function $f$ and $x \in \mathcal{M}$ satisfying the condition $\sup _{\|}\left\|\sigma_{t+i s}(x)\right\| \leq M$ for some $M>0$ uniformly $t \in \mathbb{R}$, the following $s \in[-1 / 4,1 / 4]$

(bounded) Dirichlet form $(\mathcal{E}, \mathcal{H})$ was constructed in $[3,9]$ :

$$
\begin{aligned}
& \mathcal{E}(\eta, \xi)= \int\left\langle\left[\sigma_{t-i / 4}(x)-j\left(\sigma_{t-i / 4}\left(x^{*}\right)\right)\right] \eta,\right. \\
& {\left.\left[\sigma_{t-i / 4}(x)-j\left(\sigma_{t-i / 4}\left(x^{*}\right)\right)\right] \xi\right\rangle f(t) d t } \\
&+\int\left\langle\left[\sigma_{t-i / 4}\left(x^{*}\right)-j\left(\sigma_{t-i / 4}(x)\right)\right] \eta,\right. \\
& {\left.\left[\sigma_{t-i / 4}\left(x^{*}\right)-j\left(\sigma_{t-i / 4}(x)\right)\right] \xi\right\rangle f(t) d t . }
\end{aligned}
$$

Putting $x=\frac{1}{\sqrt{2}}\left(x_{1}+i x_{2}\right), x_{1}=x_{1}^{*}, x_{2}=x_{2}^{*} \in \mathcal{M}$, the above form $(\mathcal{E}, \mathcal{H})$ can be rewritten as

$$
\begin{aligned}
& \mathcal{E}(\eta, \xi)= \int\left\langle\left[\sigma_{t-i / 4}\left(x_{1}\right)-j\left(\sigma_{t-i / 4}\left(x_{1}\right)\right)\right] \eta\right. \\
& {\left.\left[\sigma_{t-i / 4}\left(x_{1}\right)-j\left(\sigma_{t-i / 4}\left(x_{1}\right)\right)\right] \xi\right\rangle f(t) d t } \\
&+\int\left\langle\left[\sigma_{t-i / 4}\left(x_{2}\right)-j\left(\sigma_{t-i / 4}\left(x_{2}\right)\right)\right] \eta\right. \\
& {\left.\left[\sigma_{t-i / 4}\left(x_{2}\right)-j\left(\sigma_{t-i / 4}\left(x_{2}\right)\right)\right] \xi\right\rangle f(t) d t }
\end{aligned}
$$


for any $\eta, \xi \in \mathcal{H}$. Thus the (bounded) Dirichlet operator $H$ associated with $(\mathcal{E}, \mathcal{H})$ is

$$
\begin{aligned}
H= & \int\left[\sigma_{t+i / 4}\left(x_{1}\right)-j\left(\sigma_{t+i / 4}\left(x_{1}\right)\right)\right]\left[\sigma_{t-i / 4}\left(x_{1}\right)-j\left(\sigma_{t-i / 4}\left(x_{1}\right)\right)\right] f(t) d t \\
& +\int\left[\sigma_{t+i / 4}\left(x_{2}\right)-j\left(\sigma_{t+i / 4}\left(x_{2}\right)\right)\right]\left[\sigma_{t-i / 4}\left(x_{2}\right)-j\left(\sigma_{t-i / 4}\left(x_{2}\right)\right)\right] f(t) d t
\end{aligned}
$$

Notice that $j(B)=J B J \in \mathcal{M}^{\prime}$ and $J B \xi_{0}=\Delta^{1 / 2} B^{*} \xi_{0}$ for any $B \in \mathcal{M}$. Using the symmetric embedding map, define the operator $G$ on $\mathcal{M}$ given by

$$
\Delta^{1 / 4} G(A) \xi_{0}=H \Delta^{1 / 4} A \xi_{0}, A \in \mathcal{M}
$$

and the semigroup $S_{t}=e^{-t G}$ on $\mathcal{M}$. Then

$$
\begin{aligned}
G(A) & =\int G(A, t) f(t) d t \\
& \equiv \int\left[G_{1}(A, t)+G_{2}(A, t)\right] f(t) d t
\end{aligned}
$$

where

$$
\begin{aligned}
G_{1}(A, t)= & \sigma_{t+i / 2}\left(x_{1}\right) \sigma_{t}\left(x_{1}\right) A+A \sigma_{t}\left(x_{1}\right) \sigma_{t-i / 2}\left(x_{1}\right) \\
& -\sigma_{t+i / 2}\left(x_{1}\right) A \sigma_{t}\left(x_{1}\right)-\sigma_{t}\left(x_{1}\right) A \sigma_{t-i / 2}\left(x_{1}\right)
\end{aligned}
$$

and

$$
\begin{aligned}
G_{2}(A, t)= & \sigma_{t+i / 2}\left(x_{2}\right) \sigma_{t}\left(x_{2}\right) A+A \sigma_{t}\left(x_{2}\right) \sigma_{t-i / 2}\left(x_{2}\right) \\
& -\sigma_{t+i / 2}\left(x_{2}\right) A \sigma_{t}\left(x_{2}\right)-\sigma_{t}\left(x_{2}\right) A \sigma_{t-i / 2}\left(x_{2}\right) .
\end{aligned}
$$

The operator $G$ is a generator of the KMS-symmetric Markovian semigroup $\left\{S_{t}\right\}$ associated to $x \in \mathcal{M}$. See Remark 2.1 of [3] and Theorem 2.12 of [6].

\section{Invariant subspaces}

In this section we give the sufficient and necessary conditions for $x$ used to construct the semigroup $\left\{S_{t}\right\}$ in Section 2 so that the semigroup acts separately on diagonal and off-diagonal operators with respect to a basis, and study some results.

Let $\mathfrak{h}$ be a complex separable Hilbert space and let $L(\mathfrak{h})$ be the von Neumann algebra of all bounded operators on $\mathfrak{h}$. The faithful, normal, semifinite trace on $L(\mathfrak{h})$ is denoted by Tr. The Hilbert-Schmidt class $L^{2}(\mathfrak{h})$ is a Hilbert space with the inner product $\langle\xi, \eta\rangle=\operatorname{Tr}\left(\xi^{*} \eta\right)$. Consider the faithful, normal representation $\pi_{L}$ of $L(\mathfrak{h})$ given by

$$
\pi_{L}: L(\mathfrak{h}) \longrightarrow \mathcal{L}\left(L^{2}(\mathfrak{h})\right), \pi_{L}(x)=L_{x},
$$

where $L_{x}$ is the left multiplication operator, $\xi \mapsto x \xi$. Put $\mathcal{M}\left(=\mathcal{L}^{\infty}\right)=$ $\pi_{L}(L(\mathfrak{h}))$. 
The closed convex cone $L_{+}^{2}(\mathfrak{h})$ in $L^{2}(\mathfrak{h})$ consisting of nonnegative HilbertSchmidt operators is a self-dual cone in the sense that

$$
L_{+}^{2}(\mathfrak{h})=\left\{\xi \in L^{2}(\mathfrak{h}):\langle\xi, \eta\rangle \geq 0 \forall \eta \in L_{+}^{2}(\mathfrak{h})\right\} .
$$

The associated antiunitary conjugation $J$ on $L^{2}(\mathfrak{h})$ is simply the adjoint operator on $L^{2}(\mathfrak{h}): J \xi=\xi^{*}$. Then $\left(\mathcal{M}, L^{2}(\mathfrak{h}), L_{+}^{2}(\mathfrak{h}), *\right)$ is a standard form for $L(\mathfrak{h})$.

The following notation remains highly convenient. For vectors $e, f \in \mathfrak{h}$, let $|e\rangle\langle f|$ denote the operator on $\mathfrak{h}$ given by $|e\rangle\langle f| v=\langle f, v\rangle e$. Thus $|e\rangle\langle f|$ is one rank operator on $\mathfrak{h}$ and so $|e\rangle\langle f| \in L(\mathfrak{h})$.

Now let $\left\{e_{k}\right\}_{k=0}^{\infty}$ be an orthonormal basis of $\mathfrak{h}$. Let $\rho, \omega$ and $\xi_{0}$ be a strictly positive density matrix, the corresponding normal state and the corresponding cyclic and separating vector, respectively:

$$
\begin{aligned}
& \rho=\sum_{k=0}^{\infty} r_{k}\left|e_{k}\right\rangle\left\langle e_{k}\right|, r_{k}>0 \text { and } \sum_{k=0}^{\infty} r_{k}=1, \\
& \omega\left(L_{x}\right)=\operatorname{Tr}(\rho x)=\left\langle\xi_{0}, x \xi_{0}\right\rangle, \\
& \xi_{0}=\rho^{1 / 2}=\sum_{k=0}^{\infty} r_{k}^{1 / 2}\left|e_{k}\right\rangle\left\langle e_{k}\right| .
\end{aligned}
$$

The action of the associated modular operator and the modular group are given by

$$
\begin{aligned}
\Delta^{1 / 2} L_{x} \xi_{0} & =\rho^{1 / 2} x, x \in L(\mathfrak{h}), \\
\sigma_{t}\left(L_{x}\right) & =L_{\rho^{i t} x \rho^{-i t}} .
\end{aligned}
$$

Since $\pi_{L}$ is a monomorphism, we identify $\mathcal{M}=\mathcal{L}^{\infty}$ with $L(\mathfrak{h})$. We will write the above second term as $\sigma_{t}(x)=\rho^{i t} x \rho^{-i t}$.

Consider a fixed element $x \in \mathcal{M}$ such that

$$
x=\sum_{l, m \geq 0} x_{l m}\left|e_{m}\right\rangle\left\langle e_{l}\right|, x_{l m} \in \mathbb{C},
$$

and let

$$
\begin{aligned}
& x_{1}=\frac{1}{\sqrt{2}}\left(x+x^{*}\right)=\sum_{l, m \geq 0} \frac{x_{l m}+\overline{x_{m l}}}{\sqrt{2}}\left|e_{m}\right\rangle\left\langle e_{l}\left|\equiv \sum_{l, m \geq 0} y_{l m}\right| e_{m}\right\rangle\left\langle e_{l}\right|, \\
& x_{2}=\frac{1}{\sqrt{2} i}\left(x-x^{*}\right)=\sum_{l, m \geq 0} \frac{x_{l m}-\overline{x_{m l}}}{\sqrt{2} i}\left|e_{m}\right\rangle\left\langle e_{l}\left|\equiv \sum_{l, m \geq 0} z_{l m}\right| e_{m}\right\rangle\left\langle e_{l}\right| .
\end{aligned}
$$

Then $x_{1}^{*}=x_{1}, x_{2}^{*}=x_{2}$ and $x=x_{1}+i x_{2}$.

Assume that

$$
\sup _{0 \leq s \leq 1 / 2} \sum_{l, m \geq 0} r_{m}^{-s} r_{l}^{s}\left|x_{l m}\right|<\infty
$$


In case $\sup _{l} \operatorname{Card}\left(\left\{m \mid x_{l m} \neq 0\right\}\right)<\infty$, the assumption (3.2) is reduced to the condition

$$
\sup _{0 \leq s \leq 1 / 2} \sum_{l \geq 0} r_{l}^{s}\left|x_{l m}\right|<\infty .
$$

It follows from (3.2) that for all $t \in \mathbb{R}$ and $s \in[-1 / 2,1 / 2], \sigma_{t+i s}(x)$ is bounded and

$$
\begin{aligned}
\sigma_{t+i s}(x) & =\rho^{i t-s} x \rho^{-i t+s} \\
& =\sum_{l, m \geq 0} r_{m}^{i t-s} r_{l}^{-i t+s} x_{l m}\left|e_{m}\right\rangle\left\langle e_{l}\right|
\end{aligned}
$$

Lemma 3.1. For each $A=\left|e_{k}\right\rangle\left\langle e_{n}\right|$, we have the following relations: for all $t \in \mathbb{R}$,

$$
\begin{aligned}
\sigma_{t+i / 2}\left(x_{1}\right) \sigma_{t}\left(x_{1}\right) A & =\sum_{l, m \geq 0} r_{m}^{i t-1 / 2} r_{l}^{1 / 2} r_{k}^{-i t} y_{l m} y_{k l}\left|e_{m}\right\rangle\left\langle e_{n}\right| \\
A \sigma_{t}\left(x_{1}\right) \sigma_{t-i / 2}\left(x_{1}\right) & =\sum_{l, m \geq 0} r_{n}^{i t} r_{m}^{1 / 2} r_{l}^{-i t-1 / 2} y_{m n} y_{l m}\left|e_{k}\right\rangle\left\langle e_{l}\right| \\
\sigma_{t+i / 2}\left(x_{1}\right) A \sigma_{t}\left(x_{1}\right) & =\sum_{l, m \geq 0} r_{m}^{i t-1 / 2} r_{k}^{-i t+1 / 2} r_{n}^{i t} r_{l}^{-i t} y_{k m} y_{l n}\left|e_{m}\right\rangle\left\langle e_{l}\right|, \\
\sigma_{t}\left(x_{1}\right) A \sigma_{t-i / 2}\left(x_{1}\right) & =\sum_{l, m \geq 0} r_{m}^{i t} r_{k}^{-i t} r_{n}^{i t+1 / 2} r_{l}^{-i t-1 / 2} y_{k m} y_{l n}\left|e_{m}\right\rangle\left\langle e_{l}\right|
\end{aligned}
$$

and

$$
\begin{aligned}
\sigma_{t+i / 2}\left(x_{2}\right) \sigma_{t}\left(x_{2}\right) A & =\sum_{l, m \geq 0} r_{m}^{i t-1 / 2} r_{l}^{1 / 2} r_{k}^{-i t} z_{l m} z_{k l}\left|e_{m}\right\rangle\left\langle e_{n}\right|, \\
A \sigma_{t}\left(x_{2}\right) \sigma_{t-i / 2}\left(x_{2}\right) & =\sum_{l, m \geq 0} r_{n}^{i t} r_{m}^{1 / 2} r_{l}^{-i t-1 / 2} z_{m n} z_{l m}\left|e_{k}\right\rangle\left\langle e_{l}\right| \\
\sigma_{t+i / 2}\left(x_{2}\right) A \sigma_{t}\left(x_{2}\right) & =\sum_{l, m \geq 0} r_{m}^{i t-1 / 2} r_{k}^{-i t+1 / 2} r_{n}^{i t} r_{l}^{-i t} z_{k m} z_{l n}\left|e_{m}\right\rangle\left\langle e_{l}\right| \\
\sigma_{t}\left(x_{2}\right) A \sigma_{t-i / 2}\left(x_{2}\right) & =\sum_{l, m \geq 0} r_{m}^{i t} r_{k}^{-i t} r_{n}^{i t+1 / 2} r_{l}^{-i t-1 / 2} z_{k m} z_{l n}\left|e_{m}\right\rangle\left\langle e_{l}\right|
\end{aligned}
$$

Proof. This is clear from (3.3).

Applying Lemma 3.1 to (2.5), we have the concrete action of the generator $G$ :

$$
G(A)=\int G(A, t) f(t) d t, \quad A=\left|e_{k}\right\rangle\left\langle e_{n}\right|
$$


where

$$
\begin{aligned}
G(A, t)= & \sum_{l, m \geq 0} r_{m}^{i t} r_{k}^{-i t} \sqrt{\frac{r_{l}}{r_{m}}}\left(y_{l m} y_{k l}+z_{l m} z_{k l}\right)\left|e_{m}\right\rangle\left\langle e_{n}\right| \\
& +\sum_{l, m \geq 0} r_{n}^{i t} r_{l}^{-i t} \sqrt{\frac{r_{m}}{r_{l}}}\left(y_{m n} y_{l m}+z_{m n} z_{l m}\right)\left|e_{k}\right\rangle\left\langle e_{l}\right| \\
& -\sum_{l, m \geq 0} r_{m}^{i t} r_{k}^{-i t} r_{n}^{i t} r_{l}^{-i t} \sqrt{\frac{r_{k}}{r_{m}}}\left(y_{k m} y_{l n}+z_{k m} z_{l n}\right)\left|e_{m}\right\rangle\left\langle e_{l}\right| \\
& -\sum_{l, m \geq 0} r_{m}^{i t} r_{k}^{-i t} r_{n}^{i t} r_{l}^{-i t} \sqrt{\frac{r_{n}}{r_{l}}}\left(y_{k m} y_{l n}+z_{k m} z_{l n}\right)\left|e_{m}\right\rangle\left\langle e_{l}\right| \\
= & \sum_{l, m \geq 0} r_{m}^{i t} r_{k}^{-i t} \sqrt{\frac{r_{l}}{r_{m}}}\left(x_{l m} \overline{x_{l k}}+\overline{x_{m l}} x_{k l}\right)\left|e_{m}\right\rangle\left\langle e_{n}\right| \\
& +\sum_{l, m \geq 0} r_{n}^{i t} r_{l}^{-i t} \sqrt{\frac{r_{m}}{r_{l}}}\left(x_{m n} \overline{x_{m l}}+\overline{x_{n m}} x_{l m}\right)\left|e_{k}\right\rangle\left\langle e_{l}\right| \\
& -\sum_{l, m \geq 0} r_{m}^{i t} r_{k}^{-i t} r_{n}^{i t} r_{l}^{-i t}\left(\sqrt{\frac{r_{k}}{r_{m}}}+\sqrt{\frac{r_{n}}{r_{l}}}\right)\left(x_{k m} \overline{x_{n l}}+\overline{x_{m k}} x_{l n}\right)\left|e_{m}\right\rangle\left\langle e_{l}\right| .
\end{aligned}
$$

Now we fix the admissible function $f$ given by

$$
f(t)=\frac{1}{\sqrt{2 \pi}} g=\frac{1}{\pi} \int\left(e^{k / 4}+e^{-k / 4}\right)^{-1} e^{-\frac{1}{2} k^{2}} e^{-i k t} d k,
$$

where $g$ is defined as in (2.2). Notice that $g$ is the Fourier transform of $h(k)=$ $2\left(e^{k / 4}+e^{-k / 4}\right)^{-1} e^{-\frac{1}{2} k^{2}}$. Thus

$$
\begin{aligned}
\int f(t) e^{i p t} d t & =2\left(e^{p / 4}+e^{-p / 4}\right)^{-1} e^{-\frac{1}{2} p^{2}} \\
& =\operatorname{sech}\left(\frac{p}{4}\right) e^{-\frac{1}{2} p^{2}}
\end{aligned}
$$

for any $p \in \mathbb{R}$. Applying (3.5) to (3.4), the generator $G$ can be written as, for $A=\left|e_{k}\right\rangle\left\langle e_{n}\right|$,

$$
\begin{aligned}
G(A)= & \sum_{l, m \geq 0} \operatorname{sech}\left(\frac{1}{4} \ln \frac{r_{m}}{r_{k}}\right) e^{-\frac{1}{2}\left(\ln \frac{r_{m}}{r_{k}}\right)^{2}} \sqrt{\frac{r_{l}}{r_{m}}}\left(x_{l m} \overline{x_{l k}}+\overline{x_{m l}} x_{k l}\right)\left|e_{m}\right\rangle\left\langle e_{n}\right| \\
& +\sum_{l, m \geq 0} \operatorname{sech}\left(\frac{1}{4} \ln \frac{r_{n}}{r_{l}}\right) e^{-\frac{1}{2}\left(\ln \frac{r_{n}}{r_{l}}\right)^{2}} \sqrt{\frac{r_{m}}{r_{l}}}\left(x_{m n} \overline{x_{m l}}+\overline{x_{n m}} x_{l m}\right)\left|e_{k}\right\rangle\left\langle e_{l}\right| \\
& -\sum_{l, m \geq 0} \operatorname{sech}\left(\frac{1}{4} \ln \frac{r_{m} r_{n}}{r_{k} r_{l}}\right) e^{-\frac{1}{2}\left(\ln \frac{r_{m} r_{n}}{r_{k} r_{l}}\right)^{2}}\left(\sqrt{\frac{r_{k}}{r_{m}}}+\sqrt{\frac{r_{n}}{r_{l}}}\right) \\
& \times\left(x_{k m} \overline{x_{n l}}+\overline{x_{m k}} x_{l n}\right)\left|e_{m}\right\rangle\left\langle e_{l}\right| .
\end{aligned}
$$



bers:

Consider two conditions for the sequence $\left\{x_{l m}\right\}_{l, m \geq 0}$ of the complex num-

$$
\begin{aligned}
& x_{k m} \overline{x_{k l}}+\overline{x_{m k}} x_{l k}=0, \quad l \neq m, l \neq k, m \neq k, \\
& x_{m m} \overline{x_{m k}}+\overline{x_{m m}} x_{k m}=x_{k m} \overline{x_{k k}}+\overline{x_{m k}} x_{k k}, \quad m \neq k
\end{aligned}
$$

for all $k$.

Lemma 3.2. Let the sequence $\left\{x_{l m}\right\}_{l, m \geq 0}$ of the complex numbers satisfy the above condition (3.7). Then for each $k$ there exist at most three nonzero elements $x_{k l_{1}}, x_{k l_{2}}, x_{k k}, l_{1}, l_{2} \neq k$ of $\left\{x_{k m}\right\}_{m \geq 0}$ and at most nonzero three elements $x_{l_{3} k}, x_{l_{4} k}, x_{k k}, l_{3}, l_{4} \neq k$ of $\left\{x_{m k}\right\}_{m \geq 0}$.

Proof. Suppose that there exist three nonzero elements $x_{k l_{1}}, x_{k l_{2}}, x_{k l_{3}}$ such that $l_{1}, l_{2}, l_{3}(\neq k)$ are mutually different. It follows from (3.7) that we have $x_{l_{1} k}, x_{l_{2} k}, x_{l_{3} k} \neq 0$ and

$$
\begin{aligned}
& x_{k l_{1}} \overline{x_{k l_{2}}}+\overline{x_{l_{1} k}} x_{l_{2} k}=0, \\
& x_{k l_{1}} \overline{x_{k l_{3}}}+\overline{x_{l_{1} k}} x_{l_{3} k}=0, \\
& x_{k l_{2}} \overline{x_{k l_{3}}}+\overline{x_{l_{2} k}} x_{l_{3} k}=0 .
\end{aligned}
$$

Then $\frac{x_{k l_{1}}}{\overline{x_{l_{1} k}}}=-\frac{x_{l_{2} k}}{\overline{x_{k l_{2}}}}=-\frac{x_{l_{3} k}}{\overline{x_{k l}} l_{3}}, \frac{\overline{x_{l_{2} k}}}{x_{k l_{2}}}=-\frac{\overline{x_{k l_{3}}}}{x_{l_{3} k}}$, which implies $\frac{x_{l_{3} k}}{\overline{x_{k l_{3}}}}=-\frac{x_{k l_{3}}}{\overline{x_{l_{3} k} k}}$, that is, $\left|x_{l_{3} k}\right|^{2}=-\left|x_{k l_{3}}\right|^{2}$ and $x_{l_{3} k}=x_{k l_{3}}=0$. This is a contradiction. There does not exist nonzero elements $x_{k l_{1}}, x_{k l_{2}}, x_{k l_{3}}$ such that $l_{1}, l_{2}, l_{3}(\neq k)$ are mutually different.

The other part will be proved similarly.

By Lemma 3.2, rearranging the orthonormal basis $\left\{e_{k}\right\}_{k \geq 0}$, the operator $x$ satisfying (3.7) can be expressed as the following matrix form:

$$
x=\left(\begin{array}{cccccccc}
x_{00} & x_{01} & x_{02} & 0 & 0 & 0 & 0 & \cdots \\
x_{10} & x_{11} & 0 & x_{13} & 0 & 0 & 0 & \cdots \\
x_{20} & 0 & x_{22} & 0 & x_{24} & 0 & 0 & \cdots \\
0 & x_{31} & 0 & x_{33} & 0 & x_{35} & 0 & \cdots \\
0 & 0 & x_{42} & 0 & x_{44} & 0 & x_{46} & \cdots \\
0 & 0 & 0 & x_{53} & 0 & x_{55} & 0 & \cdots \\
0 & 0 & 0 & 0 & x_{64} & 0 & x_{66} & \cdots \\
. & . & . & . & . & . & . & \cdots
\end{array}\right) .
$$

We call the operator of $\mathcal{M}$ of the form $\sum_{k>0} w_{k}\left|e_{k}\right\rangle\left\langle e_{k}\right|, w_{k} \in \mathbb{C}$ the diagonal operator and $x \in \mathcal{M}$ satisfying $\operatorname{Tr}\left(x\left|e_{n}\right\rangle\left\langle e_{n}\right|\right)=0$ for all $n$ the off-diagonal operator. Let $\mathcal{M}_{d}$ be the (diagonal) subalgebra consisting of the diagonal operators and $\mathcal{M}_{\text {od }}$ the space of off-diagonal elements. Every operator in $\mathcal{M}$ is expressed as the sum of the diagonal operator and off-diagonal operator.

Theorem 3.3. Let $x=\sum_{l, m>0} x_{l m}\left|e_{m}\right\rangle\left\langle e_{l}\right|$ satisfy the assumption (3.2) and $\left\{S_{t}\right\}_{t \geq 0}$ be the semigroup with generator $G$ in (3.6) (and (3.4)). $x$ satisfies two 
conditions (3.7) and (3.8) if and only if $\mathcal{M}_{d}$ and $\mathcal{M}_{\text {od }}$ are $S_{t}$-invariant for all $t \geq 0$, that is, $S_{t}\left(\mathcal{M}_{d}\right) \subset \mathcal{M}_{d}$ and $S_{t}\left(\mathcal{M}_{o d}\right) \subset \mathcal{M}_{o d}$.

Proof. For $A=\left|e_{k}\right\rangle\left\langle e_{k}\right| \in \mathcal{M}_{d}$, we get from (3.6) that

$$
\begin{aligned}
G(A)= & \sum_{l, m \geq 0} \operatorname{sech}\left(\frac{1}{4} \ln \frac{r_{m}}{r_{k}}\right) e^{-\frac{1}{2}\left(\ln \frac{r_{m}}{r_{k}}\right)^{2}} \sqrt{\frac{r_{l}}{r_{m}}}\left(x_{l m} \overline{x_{l k}}+\overline{x_{m l}} x_{k l}\right)\left|e_{m}\right\rangle\left\langle e_{k}\right| \\
& +\sum_{l, m \geq 0} \operatorname{sech}\left(\frac{1}{4} \ln \frac{r_{k}}{r_{l}}\right) e^{-\frac{1}{2}\left(\ln \frac{r_{k}}{r_{l}}\right)^{2}} \sqrt{\frac{r_{m}}{r_{l}}}\left(x_{m k} \overline{x_{m l}}+\overline{x_{k m}} x_{l m}\right)\left|e_{k}\right\rangle\left\langle e_{l}\right| \\
& -\sum_{l, m \geq 0} \operatorname{sech}\left(\frac{1}{4} \ln \frac{r_{m}}{r_{l}}\right) e^{-\frac{1}{2}\left(\ln \frac{r_{m}}{r_{l}}\right)^{2}}\left(\sqrt{\frac{r_{k}}{r_{m}}}+\sqrt{\frac{r_{k}}{r_{l}}}\right) \\
& \times\left(x_{k m} \overline{x_{k l}}+\overline{x_{m k}} x_{l k}\right)\left|e_{m}\right\rangle\left\langle e_{l}\right| .
\end{aligned}
$$

We rewrite in detail as

$$
\begin{aligned}
& G(A) \\
& =\sum_{l \geq 0} \sqrt{\frac{r_{l}}{r_{k}}}\left(\left|x_{l k}\right|^{2}+\left|x_{k l}\right|^{2}\right)\left|e_{k}\right\rangle\left\langle e_{k}\right| \\
& +\sum_{\substack{m \neq k \\
m \geq 0}} \operatorname{sech}\left(\frac{1}{4} \ln \frac{r_{m}}{r_{k}}\right) e^{-\frac{1}{2}\left(\ln \frac{r_{m}}{r_{k}}\right)^{2}} \sum_{l \geq 0} \sqrt{\frac{r_{l}}{r_{m}}}\left(x_{l m} \overline{x_{l k}}+\overline{x_{m l}} x_{k l}\right)\left|e_{m}\right\rangle\left\langle e_{k}\right| \\
& +\sum_{m \geq 0} \sqrt{\frac{r_{m}}{r_{k}}}\left(\left|x_{m k}\right|^{2}+\left|x_{k m}\right|^{2}\right)\left|e_{k}\right\rangle\left\langle e_{k}\right| \\
& +\sum_{\substack{l \neq k \\
l \geq 0}} \operatorname{sech}\left(\frac{1}{4} \ln \frac{r_{k}}{r_{l}}\right) e^{-\frac{1}{2}\left(\ln \frac{r_{k}}{r_{l}}\right)^{2}} \sum_{m \geq 0} \sqrt{\frac{r_{m}}{r_{l}}}\left(x_{m k} \overline{x_{m l}}+\overline{x_{k m}} x_{l m}\right)\left|e_{k}\right\rangle\left\langle e_{l}\right| \\
& -2 \sum_{l \geq 0} \sqrt{\frac{r_{k}}{r_{l}}}\left(\left|x_{k l}\right|^{2}+\left|x_{l k}\right|^{2}\right)\left|e_{l}\right\rangle\left\langle e_{l}\right| \\
& -\sum_{\substack{m \neq k \\
m \geq 0}} \operatorname{sech}\left(\frac{1}{4} \ln \frac{r_{m}}{r_{k}}\right) e^{-\frac{1}{2}\left(\ln \frac{r_{m}}{r_{k}}\right)^{2}}\left(\sqrt{\frac{r_{k}}{r_{m}}}+1\right)\left(x_{k m} \overline{x_{k k}}+\overline{x_{m k}} x_{k k}\right)\left|e_{m}\right\rangle\left\langle e_{k}\right| \\
& -\sum_{\substack{l \neq k \\
l \geq 0}} \operatorname{sech}\left(\frac{1}{4} \ln \frac{r_{k}}{r_{l}}\right) e^{-\frac{1}{2}\left(\ln \frac{r_{k}}{r_{l}}\right)^{2}}\left(1+\sqrt{\frac{r_{k}}{r_{l}}}\right)\left(x_{k k} \overline{x_{k l}}+\overline{x_{k k}} x_{l k}\right)\left|e_{k}\right\rangle\left\langle e_{l}\right| \\
& -\sum_{\substack{l \neq m \\
l, m \neq k \\
l, m \geq 0}} \operatorname{sech}\left(\frac{1}{4} \ln \frac{r_{m}}{r_{l}}\right) e^{-\frac{1}{2}\left(\ln \frac{r_{m}}{r_{l}}\right)^{2}}\left(\sqrt{\frac{r_{k}}{r_{m}}}+\sqrt{\frac{r_{k}}{r_{l}}}\right) \\
& (3.10) \times\left(x_{k m} \overline{x_{k l}}+\overline{x_{m k}} x_{l k}\right)\left|e_{m}\right\rangle\left\langle e_{l}\right| .
\end{aligned}
$$


For $B=\left|e_{k}\right\rangle\left\langle e_{n}\right|, k \neq n$ in $\mathcal{M}_{o d}$, we also get

$$
\begin{aligned}
& G(B) \\
= & \operatorname{sech}\left(\frac{1}{4} \ln \frac{r_{n}}{r_{k}}\right) e^{-\frac{1}{2}\left(\ln \frac{r_{n}}{r_{k}}\right)^{2}} \sum_{l \geq 0} \sqrt{\frac{r_{l}}{r_{n}}}\left(x_{l n} \overline{x_{l k}}+\overline{x_{n l}} x_{k l}\right)\left|e_{n}\right\rangle\left\langle e_{n}\right| \\
& +\sum_{\substack{m \neq n \\
m \geq 0}} \operatorname{sech}\left(\frac{1}{4} \ln \frac{r_{m}}{r_{k}}\right) e^{-\frac{1}{2}\left(\ln \frac{r_{m}}{r_{k}}\right)^{2}} \sum_{l \geq 0} \sqrt{\frac{r_{l}}{r_{m}}}\left(x_{l m} \overline{x_{l k}}+\overline{x_{m l}} x_{k l}\right)\left|e_{m}\right\rangle\left\langle e_{n}\right| \\
& +\operatorname{sech}\left(\frac{1}{4} \ln \frac{r_{n}}{r_{k}}\right) e^{-\frac{1}{2}\left(\ln \frac{r_{n}}{r_{k}}\right)^{2}} \sum_{m \geq 0} \sqrt{\frac{r_{m}}{r_{k}}}\left(x_{m n} \overline{x_{m k}}+\overline{x_{n m}} x_{k m}\right)\left|e_{k}\right\rangle\left\langle e_{k}\right| \\
& +\sum_{\substack{l \neq k \\
l \geq 0}} \operatorname{sech}\left(\frac{1}{4} \ln \frac{r_{n}}{r_{k}}\right) e^{-\frac{1}{2}\left(\ln \frac{r_{n}}{r_{k}}\right)^{2}} \sum_{m \geq 0} \sqrt{\frac{r_{m}}{r_{l}}}\left(x_{m n} \overline{x_{m l}}+\overline{x_{n m}} x_{l m}\right)\left|e_{k}\right\rangle\left\langle e_{l}\right| \\
& -\operatorname{sech}\left(\frac{1}{4} \ln \frac{r_{n}}{r_{k}}\right) e^{-\frac{1}{2}\left(\ln \frac{r_{n}}{r_{k}}\right)^{2}} \sum_{l \geq 0}\left(\sqrt{\frac{r_{k}}{r_{l}}}+\sqrt{\frac{r_{n}}{r_{l}}}\right)\left(x_{k l} \overline{x_{n l}}+\overline{x_{l k}} x_{l n}\right)\left|e_{l}\right\rangle\left\langle e_{l}\right| \\
& -\sum_{\substack{l \neq m \\
m \geq 0}} \operatorname{sech}\left(\frac{1}{4} \ln \frac{r_{m} r_{n}}{r_{k} r_{l}}\right) e^{-\frac{1}{2}\left(\ln \frac{r_{m} r_{n}}{r_{k} r_{l}}\right)^{2}}\left(\sqrt{\frac{r_{k}}{r_{m}}}+\sqrt{\frac{r_{n}}{r_{l}}}\right) \\
\times(3.11) \quad & \left(x_{k m} \overline{x_{n l}}+\overline{x_{m k}} x_{l n}\right)\left|e_{m}\right\rangle\left\langle e_{l}\right| .
\end{aligned}
$$

By (3.10) and (3.11), $G(A) \in \mathcal{M}_{d}$ and $G(B) \in \mathcal{M}_{\text {od }}$ if and only if the following relations hold:

$$
\begin{aligned}
x_{k m} \overline{x_{k l}}+\overline{x_{m k}} x_{l k} & =0, l \neq m, l \neq k, m \neq k, \\
\sum_{l \geq 0} \sqrt{\frac{r_{l}}{r_{m}}}\left(x_{l m} \overline{x_{l k}}+\overline{x_{m l}} x_{k l}\right) & =\left(\sqrt{\frac{r_{k}}{r_{m}}}+1\right)\left(x_{k m} \overline{x_{k k}}+\overline{x_{m k}} x_{k k}\right), m \neq k, \\
\sum_{m \geq 0} \sqrt{\frac{r_{m}}{r_{l}}}\left(x_{m k} \overline{x_{m l}}+\overline{x_{k m}} x_{l m}\right) & =\left(1+\sqrt{\frac{r_{k}}{r_{l}}}\right)\left(x_{k k} \overline{x_{k l}}+\overline{x_{k k}} x_{l k}\right), l \neq k
\end{aligned}
$$

for all $k$. The above relations are reduced to (3.7) and (3.8).

In this case we have

$$
\begin{aligned}
G(A)= & 2 \sum_{l \geq 0} \sqrt{\frac{r_{l}}{r_{k}}}\left(\left|x_{l k}\right|^{2}+\left|x_{k l}\right|^{2}\right)\left|e_{k}\right\rangle\left\langle e_{k}\right| \\
& -2 \sum_{l \geq 0} \sqrt{\frac{r_{k}}{r_{l}}}\left(\left|x_{k l}\right|^{2}+\left|x_{l k}\right|^{2}\right)\left|e_{l}\right\rangle\left\langle e_{l}\right|,
\end{aligned}
$$


and

$$
\begin{aligned}
& G(B) \\
& =\sum_{\substack{m \neq n \\
m \geq 0}} \operatorname{sech}\left(\frac{1}{4} \ln \frac{r_{m}}{r_{k}}\right) e^{-\frac{1}{2}\left(\ln \frac{r_{m}}{r_{k}}\right)^{2}} \sum_{l \geq 0} \sqrt{\frac{r_{l}}{r_{m}}}\left(x_{l m} \overline{x_{l k}}+\overline{x_{m l}} x_{k l}\right)\left|e_{m}\right\rangle\left\langle e_{n}\right| \\
& +\sum_{\substack{l \neq k \\
l \geq 0}} \operatorname{sech}\left(\frac{1}{4} \ln \frac{r_{n}}{r_{k}}\right) e^{-\frac{1}{2}\left(\ln \frac{r_{n}}{r_{k}}\right)^{2}} \sum_{m \geq 0} \sqrt{\frac{r_{m}}{r_{l}}}\left(x_{m n} \overline{x_{m l}}+\overline{x_{n m}} x_{l m}\right)\left|e_{k}\right\rangle\left\langle e_{l}\right| \\
& -\sum_{\substack{l \neq m \\
m \geq 0}} \operatorname{sech}\left(\frac{1}{4} \ln \frac{r_{m} r_{n}}{r_{k} r_{l}}\right) e^{-\frac{1}{2}\left(\ln \frac{r_{m} r_{n}}{r_{k} r_{l}}\right)^{2}}\left(\sqrt{\frac{r_{k}}{r_{m}}}+\sqrt{\frac{r_{n}}{r_{l}}}\right) \\
& \quad \times\left(x_{k m} \overline{x_{n l}}+\overline{x_{m k}} x_{l n}\right)\left|e_{m}\right\rangle\left\langle e_{l}\right|,
\end{aligned}
$$

which implies $S_{t}\left(\mathcal{M}_{d}\right) \subset \mathcal{M}_{d}, S_{t}\left(\mathcal{M}_{o d}\right) \subset \mathcal{M}_{\text {od }}$. The proof is completed.

Remark 3.4. Since $\sigma_{t}(A)=A$ for all $A \in \mathcal{M}_{d}$ and $t \in \mathbb{R}$ there exists a projection ( $\sigma_{t}$-compatible conditional expectation) $P_{\mathcal{M}_{d}}$ of $\mathcal{M}$ onto $\mathcal{M}_{d}$ (Proposition 2.6.6 of [11]). The subalgebra $\mathcal{M}_{d}$ and the space $\mathcal{M}_{o d}$ are invariant for the semigroup $\left\{S_{t}\right\}_{t \geq 0}$ in Theorem 3.3. So the restriction $S_{t}^{d}:=\left.S_{t}\right|_{\mathcal{M}_{d}}$ of $S_{t}$ on $\mathcal{M}_{d}$ is also a semigroup and

$$
S_{t}^{d}\left(P_{\mathcal{M}_{d}}(A)\right)=P_{\mathcal{M}_{d}}\left(S_{t}(A)\right)
$$

for all $A \in \mathcal{M}$.

Notice that the semigroup $\left\{S_{t}\right\}_{\geq 0}$ is a KMS-symmetric semigroup on $\mathcal{M}$ (see $(2.1)$ ) and $S_{t}(\mathbf{1})=\mathbf{1}$ for all $t \geq 0$, and so $\left\{S_{t}\right\}_{t \geq 0}$ is $\rho$-invariant in the sense that

$$
\operatorname{Tr}\left(\rho S_{t}(A)\right)=\operatorname{Tr}(\rho A), A \in \mathcal{M} .
$$

Theorem 3.5. Let $\left\{S_{t}\right\}_{t \geq 0}$ be the semigroup with generator $G$ in (3.6) associate to $x \in \mathcal{M}_{d}$ as in (3.1).

(a) $S_{t}(A)=A$ for all $A \in \mathcal{M}_{d}$ and $t \geq 0$.

(b) A density matrix $\tilde{\rho}=\sum_{k \geq 0} \tilde{r_{k}}\left|e_{k}\right\rangle\left\langle e_{k}\right|$ is an invariant state for $\left\{S_{t}\right\}_{\geq 0}$, that is, $\operatorname{Tr}\left(\tilde{\rho} S_{t}(A)\right)=\operatorname{Tr}(\tilde{\rho} A)$ for all $A \in \mathcal{M}$ and all $t \geq 0$.

Proof. (a) By (3.9), we have $G(A)=0$, which is equivalent to $S_{t}(A)=A$ for all $A \in \mathcal{M}_{d}$.

(b) Let $A \in \mathcal{M}$. Then $A \in \mathcal{M}$ can be written as the sum $A=A_{d}+A_{o d}$ with $A_{d} \in \mathcal{M}_{d}$ and $A_{o d} \in \mathcal{M}_{o d}$. Since $x \in \mathcal{M}_{d}$, we get from (a) that $S_{t}\left(A_{d}\right)=A_{d}$ for all $t \geq 0$. By $S_{t}\left(A_{o d}\right) \in \mathcal{M}_{o d}$ for all $t \geq 0$, we have $\operatorname{Tr}\left(\tilde{\rho} S_{t}\left(A_{o d}\right)\right)=0$ and so

$$
\operatorname{Tr}\left(\tilde{\rho} S_{t}(A)\right)=\operatorname{Tr}\left(\tilde{\rho} S_{t}\left(A_{d}\right)\right)=\operatorname{Tr}\left(\tilde{\rho} A_{d}\right)=\operatorname{Tr}(\tilde{\rho} A)
$$

for all $t \geq 0$. The proof is completed. 


\section{References}

[1] L. Accardi, F. Fagnola, and S. Hachicha, Generic q-Markov semigroups and speed of convergence of q-algorithms, Inf. Dim. Anal. Quantum Probab. Related Topics 9 (2006), $567-594$.

[2] S. Albeverio and R. Høegh-Krohn, Dirichlet forms and Markovian semigroups on $C^{*}$ algebras, Comm. Math. Phys. 56 (1977), 173-187.

[3] C. Bahn, C. K. Ko, and Y. M. Park, Dirichlet forms and symmetric Markovian semigroups on CCR algebras with quasi-free states, J. Math. Phys. 44 (2003), 723-753.

[4] O. Bratteli and D. W. Robinson, Operator algebras and quantum statistical mechanics, Springer-Verlag, New York-Heidelberg-Berlin, vol I (1979), vol. II (1981).

[5] R. Carbone, F. Fagnola, and S. Hachicha, Generic quantum Markov semigroups: the Gaussian gauge invariant case, priprint.

[6] F. Cipriani, Dirichlet forms and Markovian semigroups on standard forms of von Neumann algebras, J. Funct. Anal. 147 (1997), 259-300.

[7] F. Cipriani, F. Fagnola, and J. M. Lindsay, Spectral Analysis and Feller Properties for Quantum Ornstein-Uhlenbeck Semigroups, Comm. Math. Phys. 210 (2000), 85-105.

[8] F. Fagnola and R. Quezada, Two-photon and emission process, Inf. Dim. Anal. Quantum Probab. Related Topics 8 (2005), 573-591.

[9] Y. M. Park, Construction of Dirichlet forms on standard forms of von Neumann algebras, Inf. Dim. Anal. Quantum Probab. Related Topics 3 (2000), 1-14.

[10] K. R. Parthasarathy, An Introduction to Quantum Stochastic Calculus, Birkhäuser, Basel, 1992

[11] V. S. Sunder, An Invitation to von Neumann Algebras, Springer-Verlag, Newyork Berlin Heidelberg London Paris Tokyo, 1986.

Veni Choi

Division of General Studies

AJou UnIVERSITY

Suwon 443-749, Korea

E-mail address: veni@ajou.ac.kr

Chul Ki Ko

UNIVERSITY COLLEGE

YONSEI UNIVERSITY

SEOul 120-749, Korea

E-mail address: kochulki@yonsei.ac.kr 